\title{
An Almost Periodic Lasota-Wazewska Dynamic Model on Time Scales
}

\section{Zhijian Yao}

Department of Mathematics and Physics, Anhui Jianzhu University, Hefei, Anhui, 230601, China e-mail: zhijianyao@126.com

\begin{abstract}
This paper deals with almost periodicity of Lasota-Wazewska dynamic equation on time scales. By applying a method based on the fixed point theorem of decreasing operator, we establish sufficient conditions for the existence of a unique almost periodic positive solution. We also give iterative sequence which converges to almost periodic positive solution. Moreover, we investigate the exponential stability of almost periodic solution by means of Gronwall inequality. Our study unifies differential and difference equations.
\end{abstract}

\section{Introduction}

As we know, biology dynamic models are very important and hot research topics. In 1976, Wazewska-Czyzewska and Lasota [1] investigated the Lasota-Wazewska model

$$
x^{\prime}(t)=-a x(t)+b e^{-\beta x(t-\tau)}
$$

which described the survival of red blood cells in animals. Kulenovic and Ladas [2] have investigated the oscillation and global attractivity of the above model. Some generalized models have been investigated by many authors, see Graef et al. [3], Kulenovic et al. [4], $\mathrm{Xu}$ and $\mathrm{Li}$ [5], Jiang and Wei [8].

In the real world, some processes vary continuously while others vary discretely. These processes can be modeled by differential equations and difference equations,

Received: November 5, 2018; Accepted: November 18, 2018

2010 Mathematics Subject Classification: 34K14, 34K20.

Keywords and phrases: Lasota-Wazewska model on time scales, exponential dichotomy, almost periodic solution, exponential stability.

Copyright (C) 2019 Zhijian Yao. This is an open access article distributed under the Creative Commons Attribution License, which permits unrestricted use, distribution, and reproduction in any medium, provided the original work is properly cited. 
respectively. However, there are also many processes that vary both continuously and discretely. Thus an interesting and challenging problem arises: How can we model these mixed processes? The theory of time scale calculus and dynamic equations on time scales provides us with a powerful tool for attacking such mixed processes. The calculus on time scales (see $[9,10]$ and references cited therein) was initiated by Stefan Hilger in his $1988 \mathrm{Ph} . \mathrm{D}$. dissertation [11] in order to unify continuous and discrete analysis, and it has a tremendous potential for applications and has recently received great attention. The two main features of the calculus on time scales are unification and extension.

The existence and stability of periodic solution or almost periodic solution for differential equations and difference equations are very basic and important problems. It is natural to ask whether we can explore such existence and stability problems in a unified way and offer more general conclusions. The study of dynamic equations on time scales can unify and extend the fields of differential and difference equations.

Motivated by the above facts, in this paper, we investigate the following nonautonomous almost periodic Lasota-Wazewska dynamic equation on time scales

$$
x^{\Delta}(t)=-a(t) x(t)+\sum_{i=1}^{n} b_{i}(t) e^{-\beta_{i}(t) x\left(t-\tau_{i}(t)\right)} .
$$

Almost periodicity is more practical and more close to the reality in biological systems $[16,17]$. In this paper, we aim to establish sufficient conditions that guarantee the existence of unique almost periodic positive solution of model (1.1). The technique used in this paper is different from the usual methods employed to solve almost periodic cases such as the contraction mapping principle and Liapunov functional. Our method is based on the fixed point theorem of decreasing operator. Particularly, we give iterative sequence which converges to the almost periodic positive solution. Moreover, we also investigate exponential stability of almost periodic positive solution by means of Gronwall inequality. The results of this paper complement and extend the previously obtained results in [2-5, 8]. Our study reveals that, for the existence and stability of almost periodic solution of differential equations and difference equations, it is unnecessary to prove results for differential equations and separately again for difference equations. We can unify such problems in the frame of dynamic equations on time scales. 


\section{Preliminaries}

In this section, we present some basic definitions and preliminary results from the calculus on time scales and almost periodic functions. For more details, see [9, 10, 14, $15]$.

The symbol $\mathbb{T}$ denotes a time scale, which is a nonempty closed subset of $\mathbb{R}$.

Definition 1. The forward and backward jump operators $\sigma, \rho: \mathbb{T} \rightarrow \mathbb{T}$ and the graininess $\mu: \mathbb{T} \rightarrow \mathbb{R}^{+}$are defined, respectively, by

$$
\sigma(t)=\inf \{s \in \mathbb{T}: s>t\}, \quad \rho(t)=\sup \{s \in \mathbb{T}: s<t\}, \quad \mu(t)=\sigma(t)-t .
$$

A point $t \in \mathbb{T}$ is called left-dense if $t>\inf \mathbb{T}$ and $\rho(t)=t$, left-scattered if $\rho(t)<t$, right-dense if $t<\sup \mathbb{T}$ and $\sigma(t)=t$, and right-scattered if $\sigma(t)>t$.

If $\mathbb{T}$ has a left-scattered maximum $m$, define $\mathbb{T}^{k}=\mathbb{T}-\{m\}$; otherwise, set $\mathbb{T}^{k}=\mathbb{T}$.

If $\mathbb{T}$ has a right-scattered minimum $m$, define $\mathbb{T}_{k}=\mathbb{T}-\{m\}$; otherwise, set $\mathbb{T}_{k}=\mathbb{T}$.

Definition 2. A function $f: \mathbb{T} \rightarrow \mathbb{R}$ is right-dense continuous provided it is continuous at right-dense points in $\mathbb{T}$ and its left-side limits exist (finite) at left-dense points in $\mathbb{T}$. If $f$ is continuous at each right-dense point and each left-dense point, then $f$ is said to be a continuous function on $\mathbb{T}$.

Definition 3. For $f: \mathbb{T} \rightarrow \mathbb{R}$, we define $f^{\Delta}(t)$ to be the number (if it exists) with the property that for any given $\varepsilon>0$, there exists a neighborhood $U$ of $t$ such that

$$
\left|(f(\sigma(t))-f(s))-f^{\Delta}(t)(\sigma(t)-s)\right|<\varepsilon|\sigma(t)-s| \quad \text { for all } s \in U .
$$

We call $f^{\Delta}(t)$ the delta (or Hilger) derivative of $f$ at $t$.

If $F^{\Delta}(t)=f(t)$, then we define the delta integral by

$$
\int_{r}^{t} f(s) \Delta s=F(t)-F(r) \quad \text { for } t, r \in \mathbb{T} .
$$

Definition 4. A function $p: \mathbb{T} \rightarrow \mathbb{R}$ is called regressive provided $1+\mu(t) p(t) \neq 0$ for all $t \in \mathbb{T}$. The set of all regressive and rd-continuous functions $p: \mathbb{T} \rightarrow \mathbb{R}$ will be denoted by $\mathfrak{R}=\mathfrak{R}(\mathbb{T}, \mathbb{R})$. 
We define the set $\mathfrak{R}^{+}=\mathfrak{R}^{+}(\mathbb{T}, \mathbb{R})=\{p \in \mathfrak{R}: 1+\mu(t) p(t)>0, \forall t \in \mathbb{T}\}$.

Definition 5. If $p$ is a regressive function, then the generalized exponential function $e_{p}$ is defined as the unique solution of the initial value problem $y^{\Delta}=p(t) y, y(s)=1$, where $s \in \mathbb{T}$.

An explicit formula for $e_{p}(t, s)$ is given by

$$
e_{p}(t, s)=\exp \left\{\int_{s}^{t} \xi_{\mu(\tau)}(p(\tau)) \Delta \tau\right\} \quad \text { for all } s, t \in \mathbb{T}
$$

with

$$
\xi_{h}(z)= \begin{cases}\frac{\log (1+h z)}{h}, & \text { if } h \neq 0, \\ z, & \text { if } h=0 .\end{cases}
$$

Definition 6. Let $p, q: \mathbb{T} \rightarrow \mathbb{R}$ are two regressive functions, define

$$
p \oplus q=p+q+\mu p q, \quad \ominus p=-\frac{p}{1+\mu p}, \quad p \ominus q=p \oplus(\ominus q) .
$$

Lemma 1. Assume that $p, q: \mathbb{T} \rightarrow \mathbb{R}$ are two regressive functions, then

(i) $e_{0}(t, s) \equiv 1, \quad e_{p}(t, t) \equiv 1$;

(ii) $e_{p}(\sigma(t), s)=(1+\mu(t) p(t)) e_{p}(t, s)$;

(iii) $\frac{1}{e_{p}(t, s)}=e_{\ominus p}(t, s), \quad e_{p}(t, s)=\frac{1}{e_{p}(s, t)}=e_{\ominus p}(s, t)$;

(iv) $e_{p}(t, s) e_{p}(s, r)=e_{p}(t, r), \quad e_{p}(t, s) e_{q}(t, s)=e_{p \oplus q}(t, s)$;

(v) $\left(e_{p}(t, s)\right)^{\Delta}=p e_{p}(t, s)$;

(vi) If $a, b, c \in \mathbb{T}$, then $\int_{a}^{b} p(s) e_{p}(c, \sigma(s)) \Delta s=e_{p}(c, a)-e_{p}(c, b)$.

Definition 7. [14] Let $\Gamma$ be a collection of sets which is constructed by subsets of $\mathbb{R}$. A time scale $\mathbb{T}$ is called an almost periodic time scale with respect to $\Gamma$, if 


$$
\Gamma^{*}=\left\{ \pm \tau \in \bigcap_{\Lambda \in \Gamma} \Lambda: t \pm \tau \in \mathbb{T}, \forall t \in \mathbb{T}\right\} \neq \varnothing
$$

and $\Gamma^{*}$ is called the smallest almost periodic set of $\mathbb{T}$.

Definition 8. [14] Let $\mathbb{T}$ be an almost periodic time scale with respect to $\Gamma$. A function $f(t) \in C\left(\mathbb{T}, \mathbb{R}^{n}\right)$ is called almost periodic if for any given $\varepsilon>0$, the set $E(f, \varepsilon)=\left\{\tau \in \Gamma^{*}:|f(t+\tau)-f(t)|<\varepsilon, \forall t \in \mathbb{T}\right\}$ is relatively dense in $\mathbb{T}$; that is, for any given $\varepsilon>0$, there exists a real number $l=l(\varepsilon)>0$ such that each interval of length $l$ contains at least one $\tau=\tau(\varepsilon) \in E(f, \varepsilon)$ satisfying $|f(t+\tau)-f(t)|<\varepsilon, \forall t \in \mathbb{T}$.

The set $E(f, \varepsilon)$ is called $\varepsilon$-translation set of $f(t), \tau$ is called $\varepsilon$-translation number of $f(t)$, and $l(\varepsilon)$ is called contain interval length of $E(f, \varepsilon)$.

Remark. If $\Gamma=\{\mathbb{R}\}$ and $\mathbb{T}=\mathbb{R}$, then $\Gamma^{*}=\mathbb{R}$, in this case, Definition 8 is equivalent to the definition of almost periodic function in [16]. If $\Gamma=\{\mathbb{Z}\}$ and $\mathbb{T}=\mathbb{Z}$, then $\Gamma^{*}=\mathbb{Z}$, in this case, Definition 8 is equivalent to the definition of almost periodic sequence in [19].

Definition 9. ([13,14]) Let $Q(t)$ be $n \times n$ rd-continuous matrix function on $\mathbb{T}$.

The linear system

$$
x^{\Delta}(t)=Q(t) x(t), \quad t \in \mathbb{T}
$$

is said to admit an exponential dichotomy on $\mathbb{T}$ if there exist positive constants $k, \alpha$, projection $P$ and the fundamental solution matrix $X(t)$ of (2.1) satisfying

$$
\begin{gathered}
\left\|X(t) P X^{-1}(\sigma(s))\right\| \leq k e_{\ominus \alpha}(t, \sigma(s)) \quad \text { for } t \geq \sigma(s), \quad s, t \in \mathbb{T}, \\
\left\|X(t)(I-P) X^{-1}(\sigma(s))\right\| \leq k e_{\ominus \alpha}(\sigma(s), t) \quad \text { for } t \leq \sigma(s), \quad s, t \in \mathbb{T} .
\end{gathered}
$$

Consider almost periodic system

$$
x^{\Delta}(t)=Q(t) x(t)+g(t), \quad t \in \mathbb{T}
$$

where $Q(t)$ is an almost periodic matrix function, $g(t)$ is an almost periodic vector function. 
Lemma 2. ([14, 15]) If the linear system (2.1) admits an exponential dichotomy, then the almost periodic system (2.2) has a unique almost periodic solution $x(t)$ as follows:

$$
x(t)=\int_{-\infty}^{t} X(t) P X^{-1}(\sigma(s)) g(s) \Delta s-\int_{t}^{+\infty} X(t)(I-P) X^{-1}(\sigma(s)) g(s) \Delta s .
$$

Lemma 3. [9] Let $Q(t)$ be a regressive $n \times n$ matrix-valued function on $\mathbb{T}$. Let $t_{0} \in \mathbb{T}$ and $x_{0} \in \mathbb{R}^{n}$. Then the initial value problem

$$
x^{\Delta}(t)=Q(t) x(t), \quad x\left(t_{0}\right)=x_{0}
$$

has a unique solution $x(t)$ as follows:

$$
x(t)=e_{Q}\left(t, t_{0}\right) x_{0} .
$$

Lemma 4. [14] Let $c_{i}(t)$ be almost periodic function on $\mathbb{T}$, where $c_{i}(t)>0$, $-c_{i}(t) \in \mathfrak{R}^{+}, \forall t \in \mathbb{T}$ and $\min _{1 \leq i \leq n}\left\{\inf _{t \in \mathbb{T}} c_{i}(t)\right\}>0$.

Then the linear system

$$
x^{\Delta}(t)=\operatorname{diag}\left(-c_{1}(t),-c_{2}(t), \ldots,-c_{n}(t)\right) x(t)
$$

admits an exponential dichotomy on $\mathbb{T}$.

By Lemma 3, we can get

Lemma 5. Let $-C=\operatorname{diag}\left(-c_{1}(t),-c_{2}(t), \ldots,-c_{n}(t)\right)$. Then $X(t)=e_{-C}\left(t, t_{0}\right)$ is a fundamental solution matrix of the linear system $x^{\Delta}(t)=\operatorname{diag}\left(-c_{1}(t),-c_{2}(t), \ldots\right.$, $\left.-c_{n}(t)\right) x(t)$.

Definition 10. Let $X$ be a Banach space and $P$ be a closed, nonempty subset of $X$. Then $P$ is called a cone if (i) $x \in P, \lambda \geq 0$ implies $\lambda x \in P$; (ii) $x \in P,-x \in P$ implies $x=\theta$. ( $\theta$ is zero element.)

Every cone $P \subset X$ induces an ordering in $X$, we define " $\leq$ " with respect to $P$ by $x \leq y$ if and only if $y-x \in P$.

Definition 11. A cone $P$ of $X$ is called normal cone if there exists a positive constant $\sigma$, such that $\|x+y\| \geq \sigma$ for any $x, y \in P,\|x\|=\|y\|=1$. 
Definition 12. Let $P$ be a cone of $X$ and $A: P \rightarrow P$ be an operator. Then $A$ is called decreasing if $\theta \leq x \leq y$ implies $A x \geq A y$.

The following fixed point theorem of decreasing operator (see [20]) is an important tool in our proofs.

\section{Lemma 6. [20] Suppose that}

(i) $P$ is normal cone of Banach space $X$, operator $A: P \rightarrow P$ is decreasing;

(ii) $A \theta>\theta, A^{2} \theta \geq \varepsilon_{0} A \theta$, where $\varepsilon_{0}>0$;

(iii) For $\forall 0<c<d<1$, there exists $\eta=\eta(c, d)>0$ such that

$$
A(\lambda x) \leq[\lambda(1+\eta)]^{-1} A x \quad \text { for } \forall c \leq \lambda \leq d \text { and } \theta<x \leq A \theta
$$

Then A has a unique positive fixed point $x^{*}>\theta$. Moreover, $\left\|x_{k}-x^{*}\right\| \rightarrow 0,(k \rightarrow \infty)$, where $x_{k}=A x_{k-1}(k=1,2, \ldots)$ for any initial $x_{0} \in P$.

Remark. In Lemma 6, the operator $A$ does not need continuity and compactness.

\section{A Unique Almost Periodic Positive Solution}

In this paper, we use notations: for any bounded function $f(t)$, we denote $\bar{f}=\sup _{t \in \mathbb{T}} f(t), \underline{f}=\inf _{t \in \mathbb{T}} f(t)$.

Throughout this paper, we assume that the bounded almost periodic functions $a(t), b_{i}(t), \beta_{i}(t), \tau_{i}(t)$ satisfy $0<\underline{a} \leq a(t) \leq \bar{a}, \quad 0<\underline{b_{i}} \leq b_{i}(t) \leq \bar{b}_{i}, \quad 0<\underline{\beta_{i}} \leq \beta_{i}(t) \leq$ $\bar{\beta}_{i}, \quad 0<\underline{\tau}_{i} \leq \tau_{i}(t) \leq \bar{\tau}_{i}(i=1,2, \ldots, n)$ and $-a(t) \in \mathfrak{R}^{+}$.

Due to biological significance, we restrict our attention to positive solutions of equation (1.1). The initial condition associated with equation (1.1) is given by

$$
x(t ; \phi)=\phi(t)>0 \quad \text { for } t \in\left[-\tau^{*}, 0\right]_{\mathbb{T}}, \quad \tau^{*}=\max _{1 \leq i \leq n}\left\{\bar{\tau}_{i}\right\}
$$

Let $X=\{w(t) \mid w \in C(\mathbb{T}, \mathbb{R}), w(t)$ is almost periodic function $\}$ with the norm $\|w\|=\sup _{t \in \mathbb{T}} w(t) \mid$, then $X$ is Banach space. 
For $w(t) \in X$, we consider equation

$$
x^{\Delta}(t)=-a(t) x(t)+\sum_{i=1}^{n} b_{i}(t) e^{-\beta_{i}(t) w\left(t-\tau_{i}(t)\right)} .
$$

Since $\inf _{t \in \mathbb{T}} a(t)=\underline{a}>0$, from Lemma 4 we know that the linear equation $x^{\Delta}(t)=-a(t) x(t)$ admits exponential dichotomy on $\mathbb{T}$.

Hence, by Lemma 2, we know that equation (3.0) has exactly one almost periodic solution:

$$
x_{w}(t)=\int_{-\infty}^{t} e_{-a}(t, \sigma(s)) \sum_{i=1}^{n} b_{i}(s) e^{-\beta_{i}(s) w\left(s-\tau_{i}(s)\right)} \Delta s .
$$

We define operator $A: X \rightarrow X$,

$$
(A w)(t)=\int_{-\infty}^{t} e_{-a}(t, \sigma(s)) \sum_{i=1}^{n} b_{i}(s) e^{-\beta_{i}(s) w\left(s-\tau_{i}(s)\right)} \Delta s, \quad w \in X
$$

Obviously, $w(t)$ is the almost periodic solution of equation (1.1) if and only if $w$ is the fixed point of operator $A$.

Define a cone $\Omega=\{w \mid w \in X, w(t) \geq 0, t \in \mathbb{T}\}$.

Let $M=\frac{1}{\underline{a}} \sum_{i=1}^{n} \overline{b_{i}}, \quad \delta=\max _{1 \leq i \leq n}\left\{\bar{\beta}_{i}\right\}$

Theorem 1. Assume that $\delta M \leq 1$. Then equation (1.1) has a unique almost periodic positive solution $w^{*}(t)$. Moreover, $\left\|w_{k}-w^{*}\right\| \rightarrow 0, \quad(k \rightarrow \infty), \quad w_{k}=A w_{k-1}$ $(k=1,2, \ldots)$ for any initial $w_{0} \in \Omega$.

Proof. Firstly, we prove that $A \Omega \subset \Omega$.

For $\forall w \in \Omega$, then

$$
(A w)(t)=\int_{-\infty}^{t} e_{-a}(t, \sigma(s)) \sum_{i=1}^{n} b_{i}(s) e^{-\beta_{i}(s) w\left(s-\tau_{i}(s)\right)} \Delta s>0 .
$$


In addition, for $\forall w \in \Omega$, we know that equation (3.0) has exactly one almost periodic solution

$$
x_{w}(t)=\int_{-\infty}^{t} e_{-a}(t, \sigma(s)) \sum_{i=1}^{n} b_{i}(s) e^{-\beta_{i}(s) w\left(s-\tau_{i}(s)\right)} \Delta s .
$$

Since $x_{w}(t)$ is almost periodic, $(A w)(t)$ is almost periodic.

This, together with (3.1), implies $A w \in \Omega$. So we have $A \Omega \subset \Omega$.

It is clear that $\Omega$ is normal cone, $A: \Omega \rightarrow \Omega$ is decreasing operator.

Now, we will show that condition (ii) of Lemma 6 is satisfied.

$$
\begin{aligned}
M=\frac{1}{\underline{a}} \sum_{i=1}^{n} \overline{b_{i}} & =\int_{-\infty}^{t} e_{-\underline{a}}(t, \sigma(s)) \sum_{i=1}^{n} \overline{b_{i}} \Delta s \geq(A \theta)(t) \\
& =\int_{-\infty}^{t} e_{-a}(t, \sigma(s)) \sum_{i=1}^{n} b_{i}(s) \Delta s \\
& \geq \int_{-\infty}^{t} e_{-\bar{a}}(t, \sigma(s)) \sum_{i=1}^{n} \underline{b_{i} \Delta s}=\frac{1}{\bar{a}} \sum_{i=1}^{n} \underline{b_{i}}>0,
\end{aligned}
$$

which implies $A \theta>\theta$.

Moreover, we have

$$
\begin{aligned}
\left(A^{2} \theta\right)(t) & =\int_{-\infty}^{t} e_{-a}(t, \sigma(s)) \sum_{i=1}^{n} b_{i}(s) e^{-\beta_{i}(s)(A \theta)\left(s-\tau_{i}(s)\right)} \Delta s \\
& \geq \int_{-\infty}^{t} e_{-a}(t, \sigma(s)) \sum_{i=1}^{n} b_{i}(s) e^{-\bar{\beta}_{i} M} \Delta s \\
& \geq e^{-\delta M} \int_{-\infty}^{t} e_{-a}(t, \sigma(s)) \sum_{i=1}^{n} b_{i}(s) \Delta s \\
& =\varepsilon_{0}(A \theta)(t)
\end{aligned}
$$

this implies $A^{2} \theta \geq \varepsilon_{0} A \theta$, here $\varepsilon_{0}=e^{-\delta M,} \delta=\max _{1 \leq i \leq n}\left\{\bar{\beta}_{i}\right\}$. 
Finally, we show that condition (iii) of Lemma 6 is satisfied.

Let $\forall 0<c<d<1$, for $\forall c \leq \lambda \leq d$ and $\theta<x \leq A \theta$, we have $0<\|x\| \leq$ $\|A \theta\| \leq M$.

$$
\begin{aligned}
A(\lambda x)(t) & =\int_{-\infty}^{t} e_{-a}(t, \sigma(s)) \sum_{i=1}^{n} b_{i}(s) e^{-\beta_{i}(s) \lambda x\left(s-\tau_{i}(s)\right)} \Delta s \\
& =\int_{-\infty}^{t} e_{-a}(t, \sigma(s)) \sum_{i=1}^{n}\left(b_{i}(s) e^{-\beta_{i}(s) x\left(s-\tau_{i}(s)\right)} e^{(1-\lambda) \beta_{i}(s) x\left(s-\tau_{i}(s)\right)}\right) \Delta s \\
& \leq \int_{-\infty}^{t} e_{-a}(t, \sigma(s)) \sum_{i=1}^{n}\left(b_{i}(s) e^{-\beta_{i}(s) x\left(s-\tau_{i}(s)\right)} e^{(1-\lambda) \bar{\beta}_{i} M}\right) \Delta s \\
& \leq e^{(1-\lambda) \delta M} \int_{-\infty}^{t} e_{-a}(t, \sigma(s)) \sum_{i=1}^{n} b_{i}(s) e^{-\beta_{i}(s) x\left(s-\tau_{i}(s)\right)} \Delta s \\
& =\frac{1}{\lambda} \cdot \lambda e^{(1-\lambda) \delta M}(A x)(t) .
\end{aligned}
$$

Let $f(t)=t e^{(1-t) \delta M}$, we have

$$
f^{\prime}(t)=e^{(1-t) \delta M}-\delta M t e^{(1-t) \delta M}=(1-\delta M t) e^{(1-t) \delta M} .
$$

Since $\delta M \leq 1$, we know $f^{\prime}(t)>0$ for $0<t<1$.

So we have $0=f(0)<f(c) \leq f(\lambda) \leq f(d)<f(1)=1$.

Hence, from (3.2) we get

$$
\begin{aligned}
A(\lambda x)(t) & \leq \frac{1}{\lambda} f(\lambda)(A x)(t) \leq \frac{1}{\lambda} f(d)(A x)(t) \\
& =\frac{1}{\lambda} \cdot \frac{1}{1+\left(\frac{1}{f(d)}-1\right)}(A x)(t)=\frac{1}{\lambda} \cdot \frac{1}{1+\eta(d)}(A x)(t)
\end{aligned}
$$

here $\eta=\eta(d)=\frac{1}{f(d)}-1>0$. 
By Lemma 6, we know operator $A$ has a unique positive fixed point $w^{*}>\theta$, which means equation (1.1) has a unique almost periodic positive solution $w^{*}(t)$. Moreover, $\left\|w_{k}-w^{*}\right\| \rightarrow 0, \quad(k \rightarrow \infty), \quad w_{k}=A w_{k-1}(k=1,2, \ldots)$ for any initial $w_{0} \in \Omega$. The proof is complete.

Remark. From the above proof, we have

$$
\begin{aligned}
w^{*}(t)=\left(A w^{*}\right)(t) & =\int_{-\infty}^{t} e_{-a}(t, \sigma(s)) \sum_{i=1}^{n} b_{i}(s) e^{-\beta_{i}(s) w^{*}\left(s-\tau_{i}(s)\right)} \Delta s \\
& \leq \int_{-\infty}^{t} e_{-a}(t, \sigma(s)) \sum_{i=1}^{n} b_{i}(s) \Delta s \\
& \leq \int_{-\infty}^{t} e_{-\underline{a}}(t, \sigma(s)) \sum_{i=1}^{n} \overline{b_{i}} \Delta s \\
& =\frac{1}{\underline{a}} \sum_{i=1}^{n} \overline{b_{i}}=M .
\end{aligned}
$$

Moreover, we also have

$$
\begin{aligned}
w^{*}(t)=\left(A w^{*}\right)(t) & =\int_{-\infty}^{t} e_{-a}(t, \sigma(s)) \sum_{i=1}^{n} b_{i}(s) e^{-\beta_{i}(s) w^{*}\left(s-\tau_{i}(s)\right)} \Delta s \\
& \geq \int_{-\infty}^{t} e_{-a}(t, \sigma(s)) \sum_{i=1}^{n} b_{i}(s) e^{-\bar{\beta}_{i} M} \Delta s \\
& \geq e^{-\delta M} \int_{-\infty}^{t} e_{-a}(t, \sigma(s)) \sum_{i=1}^{n} b_{i}(s) \Delta s \\
& \geq e^{-\delta M} \int_{-\infty}^{t} e_{-\bar{a}}(t, \sigma(s)) \sum_{i=1}^{n} \underline{b_{i}} \Delta s \\
& \geq e^{-\delta M} \frac{1}{\bar{a}} \sum_{i=1}^{n} \underline{b_{i}} .
\end{aligned}
$$


So we get

$$
e^{-\delta M} \frac{1}{\bar{a}} \sum_{i=1}^{n} \underline{b_{i}} \leq w^{*}(t) \leq M .
$$

\section{Exponential Stability}

Theorem 2. Assume that $\delta M<1$. Then equation (1.1) has a unique exponentially stable almost periodic positive solution.

Proof. Since the condition $\delta M<1$ is satisfied, by Theorem 1 we know equation (1.1) has a unique almost periodic positive solution $w^{*}(t)$, and $e^{-\delta M} \frac{1}{\bar{a}} \sum_{i=1}^{n} \underline{b}_{i} \leq w^{*}(t)$ $\leq M$. Let $\psi(t)$ be the initial function of $w^{*}(t), w^{*}(t ; \psi)=\psi(t)$ for $t \in\left[-\tau^{*}, 0\right]_{\mathbb{T}}$. Now we prove $w^{*}(t)$ is exponentially stable.

Suppose $x(t)$ is arbitrary positive solution of equation (1.1) with initial function $x(t ; \phi)=\phi(t)>0, t \in\left[-\tau^{*}, 0\right]_{\mathbb{T}}$.

Let $y(t)=x(t)-w^{*}(t)$, then we have

$$
\begin{aligned}
y^{\Delta}(t)= & \left(x(t)-w^{*}(t)\right)^{\Delta} \\
= & -a(t) x(t)+\sum_{i=1}^{n} b_{i}(t) e^{-\beta_{i}(t) x\left(t-\tau_{i}(t)\right)} \\
& -\left(-a(t) w^{*}(t)+\sum_{i=1}^{n} b_{i}(t) e^{-\beta_{i}(t) w^{*}\left(t-\tau_{i}(t)\right)}\right) \\
= & -a(t)\left(x(t)-w^{*}(t)\right)+\sum_{i=1}^{n} b_{i}(t) e^{-\beta_{i}(t) x\left(t-\tau_{i}(t)\right)} \\
& -\sum_{i=1}^{n} b_{i}(t) e^{-\beta_{i}(t) w^{*}\left(t-\tau_{i}(t)\right)} .
\end{aligned}
$$


Let

$$
g(t)=\sum_{i=1}^{n} b_{i}(t) e^{-\beta_{i}(t) x\left(t-\tau_{i}(t)\right)}-\sum_{i=1}^{n} b_{i}(t) e^{-\beta_{i}(t) w^{*}\left(t-\tau_{i}(t)\right)}
$$

Then it follows from (4.1) that

$$
y^{\Delta}(t)=-a(t) y(t)+g(t) .
$$

From (4.2), we know that $y(t)$ can be expressed as follows

$$
y(t)=e_{-a}\left(t, t_{0}\right) y\left(t_{0}\right)+\int_{t_{0}}^{t} e_{-a}(t, s) g(s) \Delta s, \quad\left(t \geq t_{0}\right), \quad t_{0} \in\left[-\tau^{*}, 0\right]_{\mathbb{T}} .
$$

Thus, (4.3) implies that

$$
y(t)=e_{-a}\left(t, t_{0}\right)\left(\phi\left(t_{0}\right)-\psi\left(t_{0}\right)\right)+\int_{t_{0}}^{t} e_{-a}(t, s) g(s) \Delta s .
$$

Note that

$$
\begin{aligned}
|g(t)| & =\left|\sum_{i=1}^{n} b_{i}(t)\left(e^{-\beta_{i}(t) x\left(t-\tau_{i}(t)\right)}-e^{-\beta_{i}(t) w^{*}\left(t-\tau_{i}(t)\right)}\right)\right| \\
& \leq \sum_{i=1}^{n} b_{i}(t)\left|e^{-\beta_{i}(t) x\left(t-\tau_{i}(t)\right)}-e^{-\beta_{i}(t) w^{*}\left(t-\tau_{i}(t)\right)}\right|
\end{aligned}
$$

By the mean value theorem, we have

$$
\begin{aligned}
\mid e^{-\beta_{i}(t) x\left(t-\tau_{i}(t)\right)}-e^{-\beta_{i}(t) w^{*}\left(t-\tau_{i}(t)\right) \mid} & =\left|-e^{-\xi}\left[\beta_{i}(t) x\left(t-\tau_{i}(t)\right)-\beta_{i}(t) w^{*}\left(t-\tau_{i}(t)\right)\right]\right| \\
& =e^{-\xi}\left|\beta_{i}(t) x\left(t-\tau_{i}(t)\right)-\beta_{i}(t) w^{*}\left(t-\tau_{i}(t)\right)\right| \\
& \leq \bar{\beta}_{i}\left|x\left(t-\tau_{i}(t)\right)-w^{*}\left(t-\tau_{i}(t)\right)\right| \\
& \leq \bar{\beta}_{i}\left\|x-w^{*}\right\| \\
& \leq \delta\left\|x-w^{*}\right\|
\end{aligned}
$$

in which $\xi$ lies between $\beta_{i}(t) x\left(t-\tau_{i}(t)\right)$ and $\beta_{i}(t) w^{*}\left(t-\tau_{i}(t)\right)$. 
Hence, by (4.5) and (4.6), we get

$$
|g(t)| \leq \sum_{i=1}^{n} b_{i}(t) \delta\left\|x-w^{*}\right\| \leq \delta\left\|x-w^{*}\right\| \sum_{i=1}^{n} \overline{b_{i}} .
$$

It follows that

$$
\|g(t)\| \leq \delta\left\|x-w^{*}\right\| \sum_{i=1}^{n} \overline{b_{i}}=\delta\|y\| \sum_{i=1}^{n} \overline{b_{i}} .
$$

Take norm at both sides of (4.4), we obtain

$$
\begin{aligned}
\|y(t)\| & \leq e_{-a}\left(t, t_{0}\right)\|\phi-\psi\|+\int_{t_{0}}^{t} e_{-a}(t, s)\|g(s)\| \Delta s \\
& \leq e_{-a}\left(t, t_{0}\right)\|\phi-\psi\|+\int_{t_{0}}^{t} e_{-a}(t, s) \delta\|y\| \sum_{i=1}^{n} \bar{b}_{i} \Delta s .
\end{aligned}
$$

From (4.7), we get

$$
\frac{\|y(t)\|}{e_{-a}\left(t, t_{0}\right)} \leq\|\phi-\psi\|+\int_{t_{0}}^{t} \frac{\|y\|}{e_{-a}\left(s, t_{0}\right)} \delta \sum_{i=1}^{n} \bar{b}_{i} \Delta s .
$$

By Gronwall inequality (see [9]), we obtain

$$
\frac{\|y(t)\|}{e_{-a}\left(t, t_{0}\right)} \leq\|\phi-\psi\| e_{\gamma}\left(t, t_{0}\right), \text { here } \gamma=\delta \sum_{i=1}^{n} \bar{b}_{i} .
$$

Hence we get

$$
\begin{aligned}
\|y(t)\| & \leq\|\phi-\psi\| e_{\gamma}\left(t, t_{0}\right) e_{-a}\left(t, t_{0}\right) \\
& \leq\|\phi-\psi\| e_{\gamma}\left(t, t_{0}\right) e_{-\underline{a}}\left(t, t_{0}\right) \\
& =\|\phi-\psi\| e_{-(\underline{a}-\gamma)}\left(t, t_{0}\right)
\end{aligned}
$$

That is

$$
\left\|x(t)-w^{*}(t)\right\| \leq\|\phi-\psi\| e_{-(\underline{a}-\gamma)}\left(t, t_{0}\right)
$$

From the condition $\delta M<1$, we know $\delta \frac{1}{\underline{a}} \sum_{i=1}^{n} \bar{b}_{i}<1$, this means $\underline{a}>\gamma$. 
Hence, (4.8) and (4.9) imply that $w^{*}(t)$ is exponentially stable. The proof is complete.

Remark. As mentioned in the introduction of this paper, one of our principal aims is to unify the existence and stability of almost periodic solution for some differential equations and their corresponding discrete analogues.

If $\mathbb{T}=\mathbb{R}$ and $\mathbb{T}=\mathbb{Z}$, then equation (1.1) reduces to

$$
x^{\prime}(t)=-a(t) x(t)+\sum_{i=1}^{n} b_{i}(t) e^{-\beta_{i}(\tau) x\left(t-\tau_{i}(t)\right)}, \quad t \in \mathbb{R}
$$

and

$$
x(k+1)-x(k)=-a(k) x(k)+\sum_{i=1}^{n} b_{i}(k) e^{-\beta_{i}(k) x\left(k-\tau_{i}(k)\right)}, \quad k \in \mathbb{Z} .
$$

Our study unifies differential equations and difference equations.

\section{Acknowledgements}

This work is supported by Natural Science Foundation of Education Department of Anhui Province (KJ2017A487).

\section{References}

[1] M. Wazewska-Czyzewska and A. Lasota, Mathematical problems of the dynamics of a system of red blood cells, Annals of Polish Mathematical Society, Series III, Applied Mathematics 6 (1976), 23-40.

[2] M. R. S. Kulenovic and G. Ladas, Linearized oscillations in population dynamics, Bull. Math. Biol. 49 (1987), 615-627.

[3] J. R. Graef, C. Qian and P.W. Spikes, Oscillation and global attractivity in a periodic delay equation, Canad. Math. Bull. 39 (1996), 275-283.

[4] M. R. S. Kulenovic, G. Ladas and Y. G. Sficas, Global attractivity in population dynamics, Comput. Math. Appl. 18 (1989), 925-928.

[5] W. Xu and J. Li, Global attractivity of the model for the survival of red blood cells with several delays, Ann. Differential Equations 14 (1998), 357-363. 
[6] Y. Kuang, Delay Differential Equations with Applications in Population Dynamics, New York: Academic Press, 1993.

[7] A. Zaghrout, A. Ammar and M. M. A. El-Sheikh, Oscillation and global attractivity in delay equation of population dynamics, Appl. Math. Comput. 77 (1996), 195-204.

[8] D. Q. Jiang and J. J. Wei, Existence of positive periodic solutions for nonautonomous delay differential equations, Chinese Ann. Math. Ser. A 20(6) (1999), 715-720.

[9] M. Bohner and A. Peterson, Dynamic Equations on Time Scales: An Introduction with Applications, Boston: Birkhäuser, 2001.

[10] M. Bohner and A. Peterson, Advances in Dynamic Equations on Time Scales, Boston: Birkhäuser, 2003.

[11] S. Hilger, Analysis on measure chains - a unified approach to continuous and discrete calculus, Results Math. 18 (1990), 18-56.

[12] R. Frankham and B. W. Brook, The importance of time scale in conservation biology and ecology, Ann. Zool. Fennici. 41 (2004), 459-463.

[13] J. Zhang, M. Fan and H. Zhu, Existence and roughness of exponential dichotomies of linear dynamic equations on time scales, Comput. Math. Appl. 59(8) (2010), 2658-2675.

[14] Y. K. Li and C. Wang, Almost periodic functions on time scales and applications, Discrete Dyn. Nat. Soc. 2011, Art. ID 727068, 20 pp. doi:10.1155/2011/727068

[15] Y. K. Li and C. Wang, Uniformly almost periodic functions and almost periodic solutions to dynamic equations on time scales, Abstr. Appl. Anal. 2011, Art. ID 341520, 22 pp. doi:10.1155/2011/341520

[16] A. Fink, Almost Periodic Differential Equations, Lecture Notes in Mathematics, vol. 377, Berlin: Springer, 1974.

[17] C. Y. He, Almost Periodic Differential Equations, Beijing: Higher Education Press, 1992.

[18] R. Yuan, On almost periodic solutions of logistic delay differential equations with almost periodic time dependence, J. Math. Anal. Appl. 330 (2007), 780-798.

[19] D. Cheban and C. Mammana, Invariant manifolds, global attractors and almost periodic solutions of nonautonomous difference equations, Nonlinear Anal. 56(4) (2004), 465484.

[20] D. Guo, Nonlinear Functional Analysis, Jinan: Shandong Science and Technology Press, 2001. 